\title{
Clinical analysis of preoperative embolization combined with Kawase approach in patients with petroclival meningioma.
}

\author{
Guozhi Song ${ }^{*}$, Shurui Li ${ }^{1}$, Xia Wang ${ }^{2}$, Cheng Chang', Jianjun Chen ${ }^{1}$, Haihong Li ${ }^{1}$, Jun Zhang ${ }^{1}$, \\ Yanyan Chao', Shen Gao $^{2}$ \\ ${ }^{1}$ Department of Neurosurgery, HanDan Central Hospital, Handan, Hebei, PR China \\ ${ }^{2}$ Department of Gynaecology and Obstetrics, HanDan Central Hospital, Handan, Hebei, PR China
}

\begin{abstract}
Objective: To investigate the effect of preoperative embolization combined with Kawase approach in the treatment of petroclival meningioma.

Methods: Retrospective analysis of 15 cases of preoperative embolization combined with Kawase approach surgical treatment of petroclival meningioma, and 15 cases of patients without preoperative embolization were treated as controls. The surgical difficulty, intraoperative blood loss and operation time of two groups were tested.

Result: The average blood loss of the preoperative embolization group was $(315.52 \pm 58.71 \mathrm{ml})$, the average blood transfusion was $(185.35 \pm 46.15 \mathrm{ml})$, the average operation time was $(2.52 \pm 0.59 \mathrm{~h})$. The mean blood loss was $(1136.25 \pm 8.26 \mathrm{ml})$, and the average blood transfusion was $(488.63 \pm 62.32 \mathrm{ml})$, the average operation time was $(5.15 \pm 1.26 \mathrm{~h})$ in the non-embolization group. The differences between the two groups were statistically significant $(P<0.05)$. The degree of tumor resection in the preoperative embolization group was significantly higher than that in the non-embolization group.

Conclusion: Preoperative embolization can significantly reduce intraoperative blood loss, operation time, and decrease the difficulty of Kawase approach surgery.
\end{abstract}

Keywords: Petroclival meningioma, Preoperative embolization, Kawase approach.

Accepted on December 12, 2017

\section{Introduction}

Meningiomas are common tumors of the central nervous system, which is located in the internal neck, and most of them are innocent tumors. Petroclival meningioma originates from dura mater which is located at the slope and the petrous apex. The treatment is very difficult due to its location that involves the central region of the basis cranii [1]. At present, exairesis is still the best treatment option for petroclival meningioma, and the extent of exairesis will directly affect the prognosis and survival time of the patients [2]. Therefore, the best choice is to remove the tumor completely, and try to avoid or reduce the incidence of postoperative complications hence, improving patients' life quality. Appropriate surgical approach is the premise of a successful resection of petroclival meningioma [3].

Preoperative embolization of the lesioned vessels and arterial blood supply arteries can reduce intraoperative hemorrhage that is conducive to tumor resection, significantly reduce the difficulty of operation thereby, bringing significant clinical effect $[4,5]$. Anterior transpetrosal approach is also called
Kawase approach which has been widely used to treat superior clivus lesions in recent years [6]. We used the preoperative superselective embolization combined with Kawase approach for resection of tumor in 15 cases at the upper slope area, and compared with 15 cases without preoperative embolization Kawase approach for resection surgery.

\section{Materials and Methods}

\section{Clinical data}

30 cases of patients with petroclival meningioma from September 2012 to September 2015 in our hospital were collected, including 12 male cases, 18 female cases. The age of patients ranged from 35 to $69 \mathrm{y}$ old with an average of (48 \pm 4.6 y). All diagnoses were confirmed by CT enhancement and MRI examination. Clinical manifestations include: 10 cases of headache and dizziness, 4 cases of limb movement disordered, 4 cases of diplopia, 9 cases that showed signs of trigeminal nerve dysfunction, 21 cases of no nerve dysfunction. 


\section{Iconography data}

All cases' diagnoses were confirmed by CT enhancement and MRI examination. Meningiomas showed an obvious enhancement in CT and MRI. The diameter of tumor was $2.8 \sim 7.5 \mathrm{~cm}$ with an average of $5.5 \mathrm{~cm}$. All cases were confirmed by postoperative pathology.

\section{Angiography and embolization}

In order to determine the necessity of superselective embolization before surgery, operators should carry on with cerebral angiography DSA petroclival meningiomas, understand the blood supply of meningioma and its relationship between peripheral vascular anatomy, and also determine whether sinus involvement or occlusion exists.

In this group, there were no vascular variation found and no dangerous anastomosis in the intracranial and extracranial arteries of the angiography of patients with petroclival meningioma. Using super catheter to the superconducting tube, target vessel that were near the tumor's vascular bed and use Glubran glue to push the embolism slowly. If the tumor was supplied by multiple branches of the external carotid artery, superselective branch embolization was embolized. If the tumor was supplied by carotid artery system, superselective tip of feeding artery was embolized. Carefully observe to exclude dangerous anastomotic branches and prevent embolic agents when entering the functional vessels.

\section{Kawase approach operation}

The operation was performed 3-5 dafter embolotherapy, and the Kawase approach was used to resect petroclival meningiomas. The lesion was located above the slope and the petroclival region, which was between the saddle back and above the internal auditory canal. This approach to Article VII, VIII cranial nerve damage was little, less affected by the sinuses sigmoid sinus and jugular bulb anatomical variation. Approach is required to maximize the removal of petrous bone but not to damage the hearing function. Its lateral is limited to the internal carotid artery and the cochlear that are separated by $2.1 \mathrm{~mm}$ thick bone.

\section{Therapeutic evaluation}

Determination of the extent of embolization: According to the standard of embolization, the embolization was divided into complete embolization, most embolization, partial embolization and small embolization.

Judgment of tumor resection degree: According to the Simpson classification standard, level 1, total resection of tumors and dural involvement, level 2, total resection of the tumor and treatment of the affected dura mater, level 3, full cut tumor but not treatment of affected dura mater, level 4, partial resection of the tumor.

\section{Statistical analysis technique}

The data were analysed by spss 19.0 software, and the experimental data were expressed by average \pm standard deviation. The two groups were compared to use t-test, comparison between groups were analysed by factor analysis of variance, count data was expressed by relative proportion $(\%)$, and were compared by chi-square criterion. Statistics was significant within $\mathrm{P}<0.05$ difference.

\section{Results}

\section{Angiography results}

Petroclival meningioma MRI showed significant enhancement. The results of MRI axial, sagittal and coronal showed that preoperative cranial MRI had a homogeneous and enhanced tumor region, but after surgery, this part of tumor area disappeared, petroclival meningioma was resected completely (Figures 1 and 2). Cerebral angiography showed that 10 patients were supplied by external carotid artery branch only, and 5 patients were combined with internal and external carotid arteries. After superselective embolization, the staining of tumor like vessels disappeared (Figure 3).

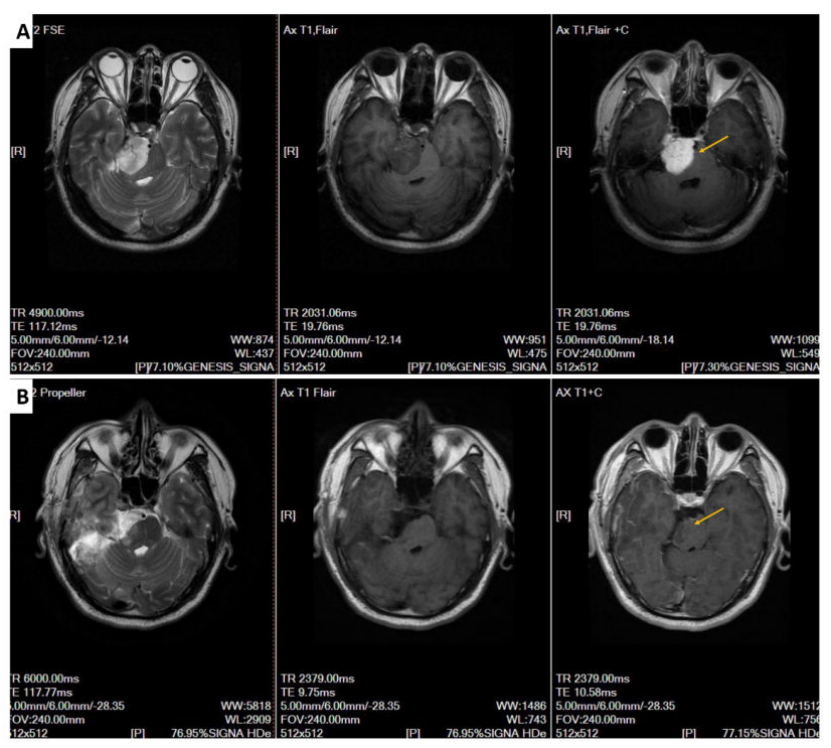

Figure 1. The cranial MRI results of petroclival meningiomas perioperatively (axial position). Note: A: the cranial MRI before operation (arrow showed the homogeneous and enhanced tumor area); B: the cranial MRI after surgery (arrow showed the complete resection of petroclival meningiomas).

\section{The effect of preoperative embolization}

According to the Bendszus evaluation standard of embolization degree, result displayed: 3 cases of complete embolization, 7 cases of most embolization, 7 cases of partial embolization, and 1 case of small embolization. The results of Kawase approach surgery showed that the amount of bleeding, blood transfusion volume and operation time of the Kawase approach group were lower than those of the other embolization group, 
and the difference was statistically significant $(\mathrm{P}<0.05$, Table $1)$.

\section{Exairesis evaluation of Kawase approach}

Evaluation of tumor resection degree: For patients with preoperative embolization treatment, 11 cases of operation degree Simpson 1 was significantly higher than the noembolization group patients, for patients with preoperative embolization treatment, 4 cases of operation degree Simpson 2 and 3 was significantly lower than the no-embolization group patients, and the difference was statistically significant $(\mathrm{P}<0.05)$ (Table 2).

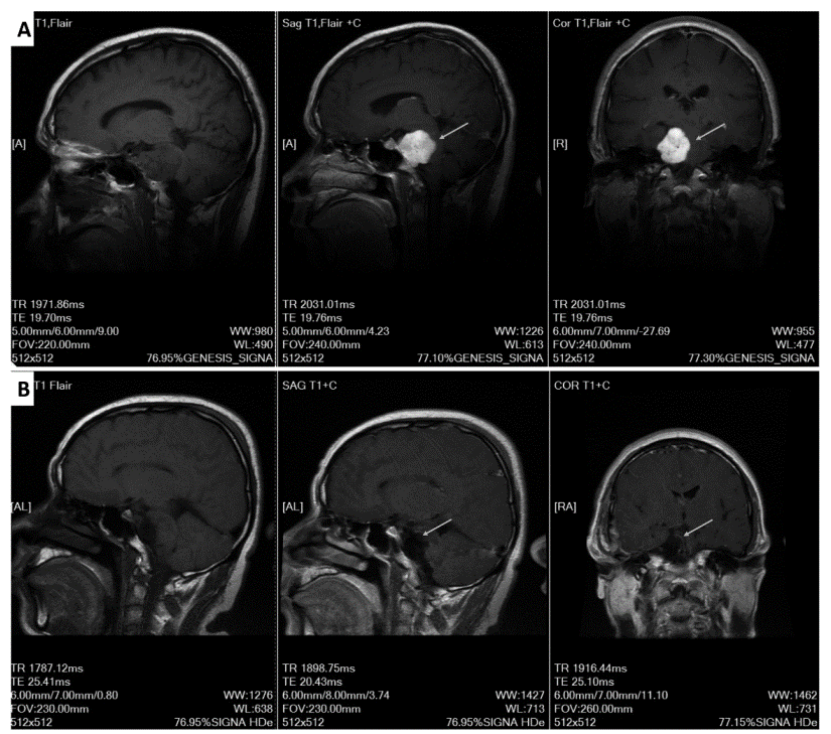

Figure 2. The cranial MRI results of petroclival meningiomas perioperatively (sagittal and coronal). Note: A: the cranial MRI before operation (arrow showed the homogeneous and enhanced tumor area); B: the cranial MRI after surgery (arrow showed the complete resection of petroclival meningiomas).

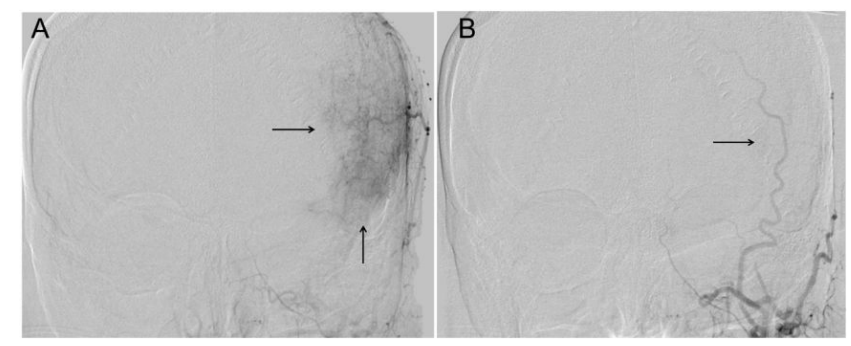

Figure 3. DSA results of whole brain angiography. Note: A: Angiographic findings before embolization (arrows showed appearance of vessels tumor like); B: Results of cerebral angiography after embolization (disappearance of the staining of tumor like vessels).

\section{Complication}

All of complete embolization, most embolization, partial embolization and small embolization group had no serious complications. There were 8 cases of scalp heat pain, but got improved after treatment. There were no complications such as intracranial hemorrhage or hemiplegic paralysis after operation.

\section{Discussion}

Meningioma is common tumor of the central nervous system, which is located in the internal neck, and it is innocent tumor in most cases. Petroclival meningioma originates from dura mater that is located at the slope and the petrous apex. The treatment is very difficult due to its location that involved the central region of the basis cranii. At present, exairesis is still the best treatment option for petroclival meningioma, and the extent of exairesis will directly affect the prognosis and survival time of the patients [7]. Therefore, the best choice is to remove the tumor completely, and try to avoid or reduce the incidence of postoperative complications hence, improving patients' life quality. Abundant blood supply-intraoperative bleeding often occurs, which brings great difficulty and risk to surgical operation. Preoperative embolization of the lesioned vessels and arterial blood supply arteries can reduce intraoperative bleeding, which is conducive for tumor resection, significantly reduce the difficulty of operation, and brings significant clinical effect $[8,9]$.

Application of small injury and good exposure surgical approach is an important factor to a successful operation. The Kawase approach also called anterior transpetrosal approach, originally introduced by Japanese scholar Kawase, later it was modified by other scholars and now it was widely used to treat upper clivus lesions [10]. Kawase approach applies to tumor at the base of skull, such as acoustic nerve tumor, margaroid tumor, meningioma and jugular vein aneurysm, etc. The advantages include; it can directly access to the prepontine cistern and euthyphoria perforating artery of pons without pulling the cerebellum, brainstem and cranial nerves, and has large operating space [11].

Using superselective preoperative embolization combined with Kawase approach for the treatment of petroclival meningioma has the following advantages. First of all, the micro catheter is near the tumor and cerebral angiography can help to deeply understand the risk of intracranial and extracranial dangerous anastomotic branches and the choice of glubran glue and lipiodol embolization agent is safe and effective, and it can avoid error thrombus. Secondly, through the preoperative embolization, Kawase approach surgery can grind a range of enlarged petrous apex bone, increasing the exposure degree of the field. But the petrous part of temporal bone is provided with cochlear, pars petrosa arteriae carotidis internae and the facial nerve and other important structures. Therefore the operator should be very familiar with these anatomical structures on the clinical operation in order to avoid intraoperative labyrinthine injury, rupture of internal carotid artery and injury of trochlear, facial, trigeminal nerve, and other complications, and it can greatly improve the total resection rate of the tumor [12]. The results of this study showed that petroclival meningiomas with Kawase approach embolization before surgery could reduce bleeding and blood 
transfusion during operation and shorten the operation time observably.

The complications of preoperative embolization of meningioma are very low. Complications include: scalp necrosis, cranial nerve palsies, cerebral infarction, encephaledema, etc. [13]. For example, cerebral infarction mostly because of the complication that is caused by the embolism of dangerous anastomotic branches. In order to ensure the quality of preoperative embolization and reduce thrombotic complications, operators need to choose the appropriate embolism method. The common embolic materials are Glubran glue, gelfoam particles, PVA particles, etc. [14]. This study used Glubran glue and lipiodol embolization agent, which have good safety and radiopacity. Operators are required to have extensive surgical experience, determined no definite dangerous anastomotic branch, and then perform further embolization treatment. In this group of patients, there were no complications such as scalp necrosis, cerebral palsy and cerebral infarction found.

To sum up, through retrospective comparative analysis, we found that embolization of petroclival meningiomas before Kawase approach operation could reduce the amount of bleeding during operation, the difficulty of operation, and shorten the operation time. Using superselective embolization was safe and reliable with no serious complications and sequelae. Therefore, for the Kawase surgical approach to petroclival meningiomas, preoperative superselective embolization can be used as an auxiliary measure in the operation.

Table 1. Comparison of surgical data between the two groups $(\bar{x} \pm s)$.

\begin{tabular}{|c|c|c|c|c|}
\hline Group & Cases & Mean bleeding volume (ml) & Mean blood transfusion (ml) & Operation time (h) \\
\hline Preoperative embolization group & 15 & $315.52 \pm 58.71^{*}$ & $185.35 \pm 46.15^{\star}$ & $2.52 \pm 0.59^{\star}$ \\
\hline No-embolization group & 15 & $1136.25 \pm 82.16$ & $488.63 \pm 62.32$ & $5.15 \pm 1.26$ \\
\hline
\end{tabular}

Note: Compared with no-embolization group: ${ }^{*} \mathrm{P}<0.05$

Table 2. Comparison of two groups of patients with tumor resection extent.

\begin{tabular}{lllll}
\hline Group & Cases & $\begin{array}{l}\text { Degree Simpson } \\
\mathbf{1}\end{array}$ & $\begin{array}{l}\text { Degree } \\
\mathbf{2 \sim 3}\end{array}$ & Simpson \\
\hline $\begin{array}{l}\text { Preoperative embolization } \\
\text { group }\end{array}$ & 15 & $11(11 / 15)$ & $4(4 / 15)$ \\
\hline No-embolization Group & 15 & $2(2 / 15)$ & $13(13 / 15)$ \\
\hline $\mathrm{X}^{2}$ & 11 & & \\
\hline $\mathrm{P}$ & $<0.001$ & & \\
\hline
\end{tabular}

\section{References}

1. Hunter JB, Weaver KD, Thompson RC. Petroclival meningiomas. Otolaryngol Clin North Am 2015; 48: 477-490.

2. Pirayesh A, Petrakakis I, Raab P. Petroclival meningiomas: Magnetic resonance imaging factors predict tumor resectability and clinical outcome. Clin Neurol Neurosurg 2016; 147: 90-97.

3. Morisako H, Goto T, Ohata K. Petroclival meningiomas resected via a combined transpetrosal approach: surgical outcomes in 60 cases and a new scoring system for clinical evaluation. J Neurosurg 2015; 122: 373-380.

4. Hanak BW, Haussen DC, Ambekar S. Preoperative embolization of intracranial hemangiopericytomas: case series and introduction of the transtumoral embolization technique. J Neurointerv Surg 2016; 8: 1084-1094.

5. Fusco MR, Salem MM, Gross BA. Preoperative embolization of extra-axial hypervascular tumors with onyx. J Cerebrovasc Endovasc Neurosurg 2016; 18: 12-18.
6. Shibao S, Borghei-Razavi H, Orii M. Anterior transpetrosal approach combined with partial posterior petrosectomy for petroclival meningiomas with posterior extension. World Neurosurg 2015; 84: 574-579.

7. Chen LH, Yang Y, Wei Q. Microsurgical management of petroclival meningiomas combined trans-subtemporal and suboccipital retrosigmoid keyhole approach. Beijing Da Xue Xue Bao 2016; 48: 738-742.

8. Ishikawa M, Yamagami T, Kakizawa H. Preoperative coil embolization in patients with a replaced hepatic artery scheduled for pancreatectomy with splanchnic artery resection helps to prevent ischemic organ injury. J Comput Assist Tomogr 2016; 40: 172-176.

9. Imai T, Ohshima T, Nishizawa T. Successful preoperative endovascular embolization of an extreme hypervascular glioblastoma mimicking an arteriovenous malformation. World Neurosurg 2016; 86: 511-514.

10. Kawase T, Shiobara R, Toya S. Middle fossa transpetrosaltranstentorial approaches for petroclival meningiomas. Selective pyramid resection and radicality. Acta Neurochir (Wien) 1994; 129: 113-120.

11. Panigrahi M, Vooturi S, Patibandla MR. Novel classification for surgical approach of petroclival meningiomas: A single-surgeon experience. Neurol India 2015; 63: 718-722.

12. Li D, Tang J, Ren C. Surgical management of medium and large petroclival meningiomas: a single institutions experience of 199 cases with long-term follow-up. Acta Neurochir (Wien) 2016; 158: 409-425.

13. Li D, Hao SY, Wang L. Recurrent petroclival meningiomas: clinical characteristics, management, and outcomes. Neurosurg Rev 2015; 38: 71-86. 
Clinical analysis of preoperative embolization combined with Kawase approach in patients with petroclival meningioma

14. Nakanishi Y, Kojima F, Kamo M. Preoperative arterial embolization with n-butyl-2 cyanoacrylate for chronic cavitary pulmonary aspergillosis with trauma induced type diabetes mellitus. Kyobu Geka 2016; 69: 184-187.

\section{*Correspondence to}

Guozhi Song

Department of Neurosurgery

HanDan Central Hospital

PR China

Email: yingcong_xiao666@yeah.net 